\title{
Neural Network Controller for Tunable Liquid Crystal Photonic Device work as Laser Beam Steering Device
}

\author{
Hayder Qassem Mashri \\ Materials Engineering Department \\ College of Engineering, Basrah University \\ Basrah, IRAQ
}

\begin{abstract}
In this paper, artificial neural network controller (NNC) was designed and used to adjust and control the work of the laser beam steering device consist of liquid crystals cell and other optical components. The target of the neurocontroller is to guarantee smooth deliver and exactly control the laser beam to a required location via control the liquid crystals cell and the optical components. Back propagation method is used to build up the neurocontroller, main artificial neurocontroller software is consists of three sub programs according to the three functions it proposed to administer. Trial results obtained after the execution of the neurocontroller affirmed the optimum performs in administrating, controlling and standardize the device while the value of the errors reached its minima, which coincidence with the approaches of the paper goals.
\end{abstract}

\section{Keyword}

Neural network; neurocontroller; Laser beam steering.

\section{INTRODUCTION}

In numerous applications, the laser beam steering is a vital topic for free-space communications, military applications, optical interconnects, projection displays, and other common en gineering applications $[1,2]$.

Many techniques have been used to achieve the laser steering such as reflect the light via without thinking controlled mirrors, but for the reason that the character of the mechanical progress, the system has a imperfect speed. It is constantly attractive to develop compact and lightweight non-mechanical beam steering devices which can compensate the big and bulky mechanical systems. Patterned liquid crystals [3] have been investigated for building small, ultra-light, swiftly steered laser beam subsystems [4]. Liquid crystals are intermediate phases between crystalline solids and isotropic liquids with common properties of both [5], that is it can work as a solid while it has liquid appearances; they are orientationally ordered fluids with anisotropic properties and electrical field sensitive. Laser beam steering as well as the liquid crystals cell was semi-manually controlled.

Liquid crystals electro-optic beam-steering devices can be classified into two types: prismatic and diffractive, a usual simple grating composition produces several diffraction orders (first or higher orders). Theoretically, diffraction efficiency of the first-order beam is about $34 \%$. The laser holographic blazing method is able to groove the grating to concentrate the light to the first order, greatly brighter spectrum is result. $100 \%$ diffraction efficiency can be reach by well-designed liquid crystals prism grating for a firstorder beam, uncomplicated technique for fabricating liquid crystals blazed gratings is to use a glass substrate with a sawtooth surface structure, as Figure 1 shows. The thickness of liquid crystals layer in this structure varies at regular intervals and asymmetrically. The sawtooth substrate is a poly methyl methacrylate (PMMA) blazed grating made by electron beam lithography. The refractive index of PMMA is mostly coincidence with the ordinary index of the selected liquid crystals, but smaller than the extraordinary index. Consequently, in the voltage off state, the in-plane distribution of the optical path length of the incident light has a sawtooth profile [4].

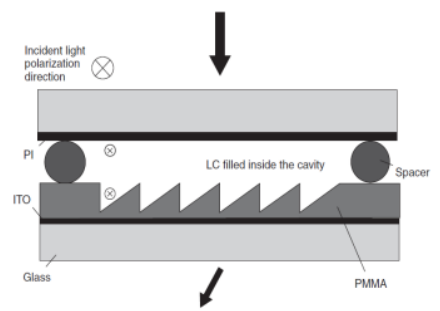

Figure1. Liquid crystals blazed-grating beam defector using a glass substrate with a sawtooth surface structure: polyimide; indium-tin-oxide; poly methyl methacrylate

Laser beam steerer can be classified into two types:

\subsection{Optical phased array}

This type of steerer composed of a one-dimensional (1-D) array of equal-spaced liquid crystals phase shifters, Fig2.

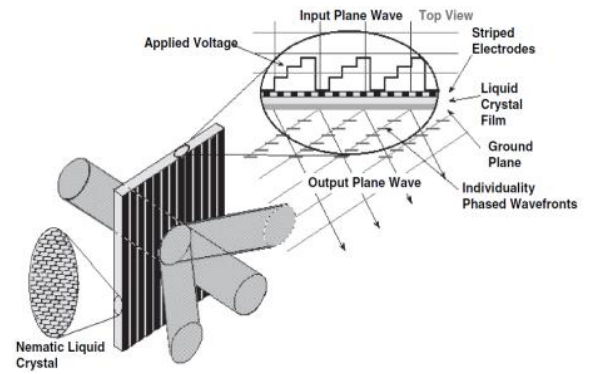

Figure2. S chematic drawing of 1-Dimension optical phased array and a conceptual imposed phase shift.

\subsection{Pris m-based beam steering}

It is a two-dimensional (2-D) prism-based tool using a birefringent prism with wedge angle $\alpha$ and a switchable 90_ Twisted Nematic liquid crystals (type of liquid crystals 
used widely in communication and display) cell as a polarization rotator, Fig3.

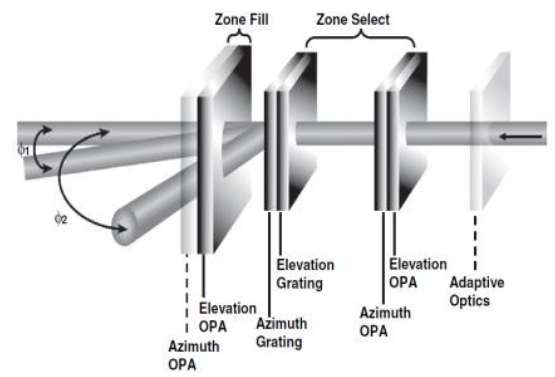

Figure 3. A 2-Dimensions beam steerer using zone select and zone fill optical phased arrays in conjunction with two gratings.

Fig4 shows the arrangement of the device and the functional mechanisms of the birefringent/isotropic biprism [4].

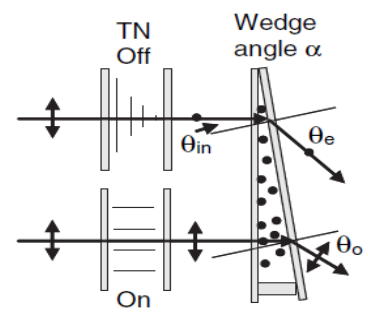

Figure 4: The functional principles of a prism-type beam-steering device.

\section{NEURAL NETWORKS}

Neural networks are structured by simulating human thinking, brain, and neural cells. It have a number of artificial neurons to do learning, memory, recalling and parallel computing, and a differentiated numerical model summaries that forecast processing of data in the future [6]. Neural network procedure has received much interest in the field of control; they have show significant enhanced results when controlling complex non-linear system than common methods, the majority of neural network controllers are approximately built by passive learning methods whereby previously trained neural network is expected to perform frequently with the same accuracy on fresh sets of data [7].

The neurocontroller can dexterously learning from the data that include information about the device parameters and to produce control performance depending on these data. Two central aspects are essential in the design of the NNC:

\section{1-Identification of the device parameters. \\ 2- Controller Design.}

Neural networks method effectively applied to both points above [8]. Many researchers applied neural networks to the laser and liquid crystals, neural networks showed good performance in the process control, for example; Smith uses neural network models to inspect changes of processing means and variations. The processing features include original data, sample mean, range and standard deviation of a sample set [9]. D. De Groff et al used liquid crystals itself as a model for neural networks [10]. Kuratomi et al applied neural networks to control liquid crystal display [11].

\subsection{Back Propagation Network (BPN)}

Back Propagation Network (BPN) model is a representative of the neural network models. The application of this model is successful on diagnosis, prediction and classification. Generalized delta rule of learning is used by the BPN model. This rule applies descent algorithm to get most small squared sum for adjusted weights. When BPN model is learning, input data is processed that feed-forward calculated difference of the network output and expected output, and feed-back adjust weights to the network. Learning cycles are recurring on the neural network until it gains convergence [12].

\section{NEURAL CONTROLLER (NEUROCONTROLLER)}

Neurocontroller is a software controlling system; its application depends on the hardware equipments, signals from the hardware are bused to the interface that transforms them to the computer as input data where the neural network program processing them. Neurocontroller most receive exact data in order to give accurate orders to the system.

The operations concerned in training the neural network are generation and validation of training on suggested data sets of the laser steering device, preprocessing of data sets, with training in addition to validation of the neural network [13]. The Neurocontroller is able to deal with input readings from laser steering device to response instantly and route orders for control and regulate the operating of the laser steering device. The performance of the neurocontroller system was depend on the simulated data that are used to train the network. Amount and distribution of data upon possibilities have a large effect on the capability of the network to control and generalize [14]. In this research signals and input data equal to the real values are produced virtually by the neural network to simulate the real work of the components of the device. Neurocontroller trained to work according to the parameters of the optimum specifications.

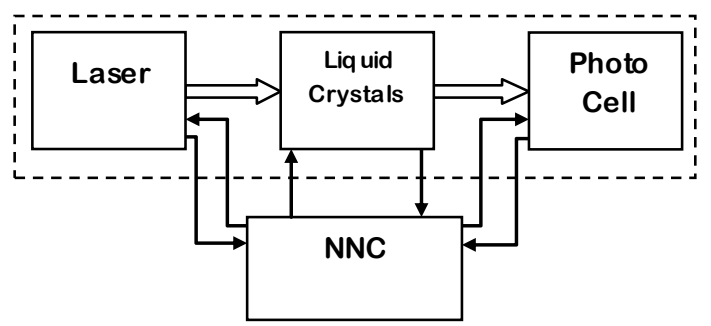

Figure5. Diagram of Laser steering device and the Neurocontroller.

\section{RESULTS AND DISSCUSION}

\subsection{Neurocontrol the Laser Beam}

Neural network controlling the laser production as: -switch on/off

-power

-modulation

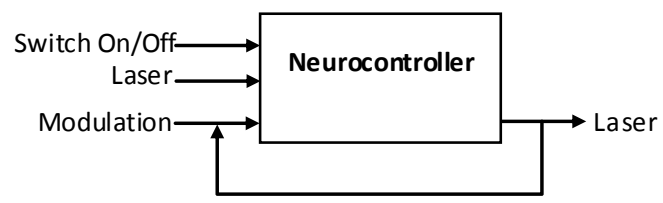

Figure6. Neurocontroller for laser production. 
Neurocontroller showed good administrating behaviour on the laser by controlling the power on/off and modulation to appropriate values.

The neurocontroller achieve proper control style, minimum error value less than 0.001 obtained after 600 tries of neurocontroller training, Fig7.

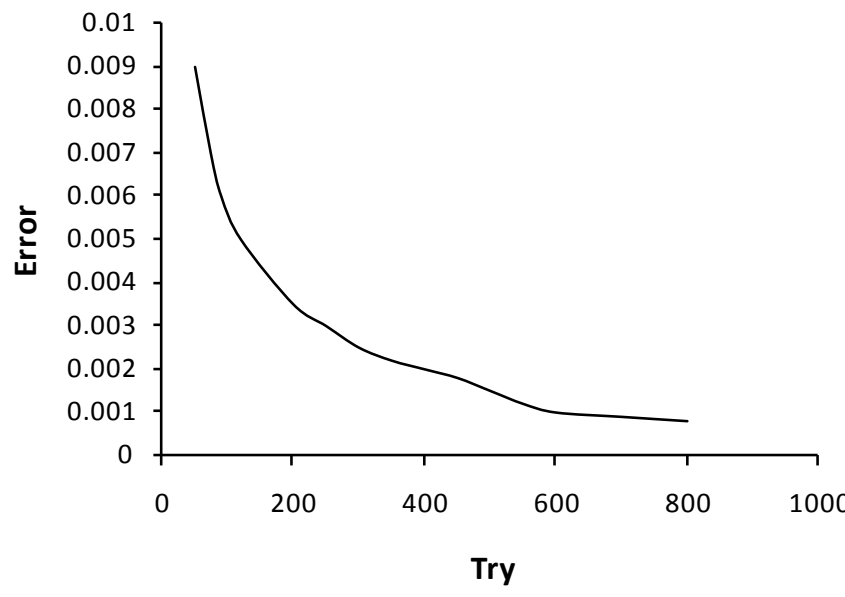

Figure7. The style of neurocontroller when keeping steady production of laser, minimum error value obtained

after 600 tries of neurocontroller training.

\subsection{Neurocontrol the Laser Beam Steerer}

Neural network, Fig8, administrate according to the following information: when the applied voltage between any given striped electrode and the underlying ground plane exceeds the Freedericksz transition threshold, the phase shifter in the liquid crystals volume underlying the patterned electrode will be created.

\subsubsection{Neurocontrol the orientation of the liquid crystals}

The degree of nematic liquid crystals reorientation depends on the applied voltage.

NNC will work according to;

1-Control the applied voltage to each pixel, a stair-step blazed phase grating can be generated [15]

2-Control the application of a periodic sequence of staircase voltage ramps of period $\mathrm{L}$ across the array aperture to create a corresponding periodic staircase profile of phase, as shown in the long-drawn-out section of Figure 2, with properly controlled weighted voltage steps, chosen to compensate for the non-linear phase-voltage profile of a typical liquid crystals, a linearly increasing phase profile will be produced.

3-If the maximal phase shift on each staircase ramp of $\mathrm{N}$ voltage steps is $2 \pi(\mathrm{N}-1) / \mathrm{N}$, NNC will adjust the periodic (modulo $2 \pi$ ) phase profile to be equivalent to a single staircase phase ramp across the aperture.

4-Adjustable prism simulated, and the beam is steered to a new direction $\theta$ (relative to the phased array boresite) given by the general grating equation:

$$
\sin \theta+\sin \theta_{\text {inc }}=\frac{\lambda_{4}}{\Lambda}
$$

Where $\theta_{\text {inc }}$ is the incident angle of the beam, and $\Lambda$ is the period of the programmed grating.

\subsubsection{Neurocontrol the steering direction}

The steering direction depends on the periodicity (and sign) of the applied voltage ramp. The diffraction efficiency $\eta$ of a grating with a stair-step blaze designed to maximize energy to first order is related to the number of steps $\mathrm{N}$. The efficiency grows rapidly with $\mathrm{N}$ and reaches _95\% as:

$$
\eta=[\sin (\pi / N) /(\pi / N)]^{2}
$$

The controlled blazed grating is switched off when the applied voltage is sufficiently high.

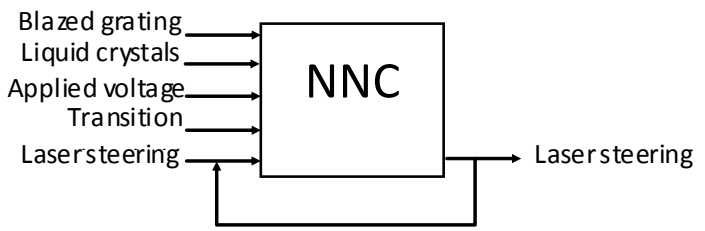

Figure8. Neurocontroller for laser beam steering.

The neurocontroller network was trained using the all above states, Fig8. General and optimum result was found after 1100 training cycles where error is reach value less than 0.0009 . Fig. 9 shows the performance of the general neurocontroller operation.

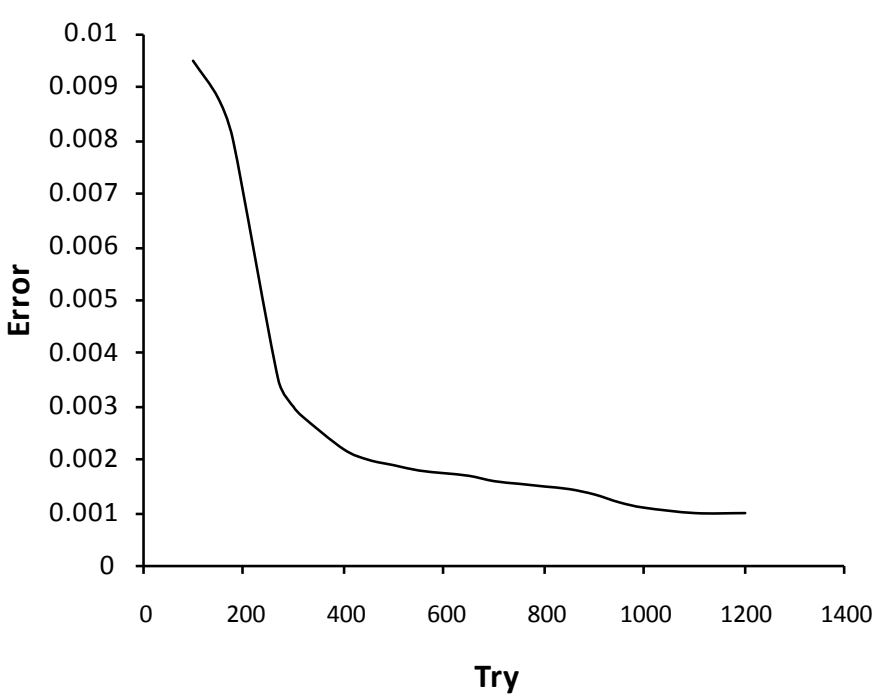

Figure9. The style of neurocontroller when laser beam steering, minimum error value obtained after 1100 tries of neurocontroller training.

\subsection{Neurocontrol the photocell}

Neurocontroller will monitor the laser beam delivery and its quality, Fig10.

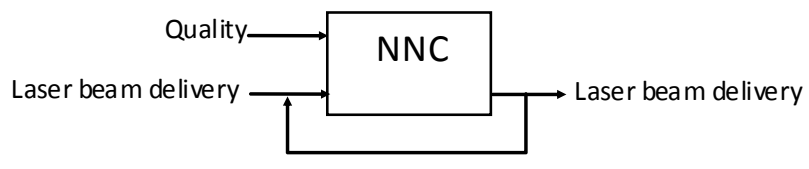

Figure 10. Neurocontroller for laser beam delivery.

The neural network trained according to standard values. The style of neurocontroller when monitoring the laser beam delivery with minimum error value of less than 0.0005 obtained after 900 tries of neurocontroller training, Fig11. 


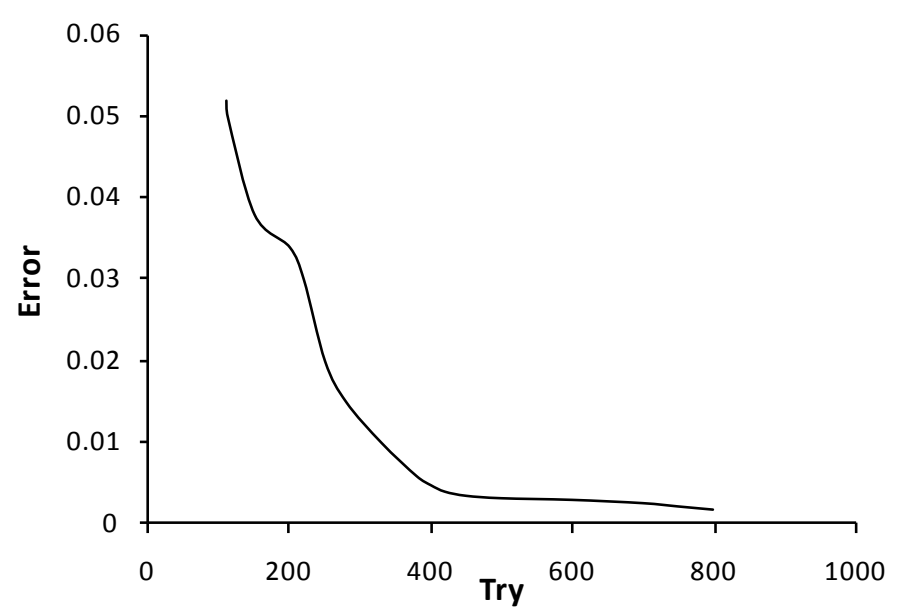

Figure11. The style of neurocontroller in laser beam delivery, minimum error value obtained after 700 tries of neurocontroller training.

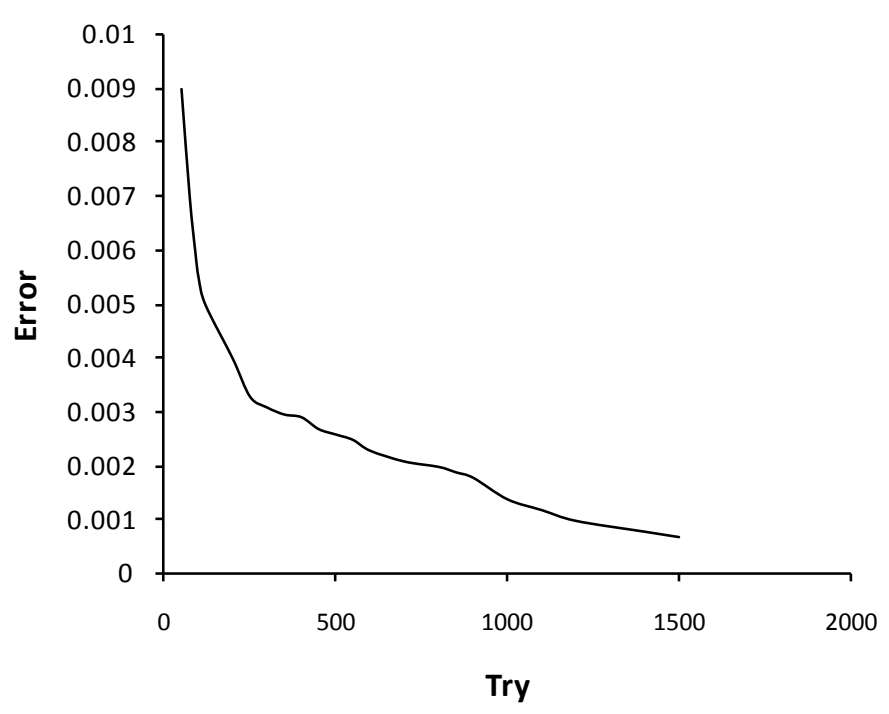

Figure 12. The overall style of neurocontroller administrating and supervising, minimum error value obtained after 1400 tries of neurocontroller training.

\section{CONCLUSION AND FUTURE WORK}

The operation of neural network controller of the laser beam steering is accurately executed in the research of this paper which represents the innovation of the research. The neural networks used the concept of task decomposition, by using the virtual standard inputs as reference, this research showed that the network take its right decisions depending on the desired right data. The neurocontroller decisions are supervise and control the laser beam and laser beam steering operation to ensure that it's operating accurately.

The experimental result showed that the neurocontroller system would be high precise, where the mean error of it reached 0.001 when controlling laser and less than 0.0009 when controlling laser beam steering, respectively. This error would be reached 0.0005 when controlling laser beam delivery. Finally, the experiment showed that the error for general neurocontroller system was less than 0.0008 , Fig12. These results with such errors fulfil that the neurocontroller have high optimization with any events in control and supervise of the laser beam steering. Future work will be the use of this neurocontroller software with real hardware.

\section{REFENCES}

[1] John F. Ready, 1997, "Industrial Applications of Lasers", 2nd ed, Academic Press Limited, ISBN 012-583961-8

[2] William M. Steen, 1991, "Laser Material Processing", Springer-Verlag, ISBN 3-540-19670-6 SpringerVerlag Berlin Heidelberg New York

[3] D. S. Wiersma and S. Cavalieri, 2002, Temperaturecontrolled random laser action in liquid crystal infiltrated sy stems, Phy sical Review E 66, 056612

[4] D. K. Yang and S. T. Wu, 2006, "Fundamentals of Liquid Crystal Devices", John Wiley \& Sons, Ltd. ISBN: 0-470-01542-X

[5] M. Barón and R. F. T. Stepto, 2002, "Definitions Of Basic Terms Relating To Polymer Liquid Crystals", Pure Appl. Chem., Vol. 74, No. 3, pp. 493-509.

[6] G. Dreyfus, 2005, "Neural Networks Methodology and Applications", Springer-Verlag Berlin Heidelberg, ISBN-10 3-540-22980-9 Springer Berlin Heidelberg New York

[7] M. Schmidt, T. Stidsen, 1997, "Hybrid Systems: Genetic Algorithms, Neural Networks, and Fuzzy Logic”, Diami IR, ISSN 0106-9969

[8] T. R. Chaudhuri, L. G. C. Hamey, R. D. Bell, 1995, Neural Network Control Using Active Learning", Control, Vol2, pp. 396-373

[9] H. LIN and C. Chien, 2002, Applying Ant Colony Algorithm and Neural Network Model to Colour Deviation Defect Detection in Liquid Crystal Displays, Systemics, Cybernetics And Informatics, Vol3,No3, pp 73-78

[10] D. De Groff , P. S. Neelakanta, R. Sudhakar and F. Medina, 1993, A liquid Crystal Model for Neural Networks, Complex Systems, Vol.7, pp43-57

[11] Kuratomi etal, 1996, Neural network device and image recognition method employing photoconductive liquid crystal device with patterned electrode, Patent Number 5515189

[12] X. Hu, 1995, Dynamic Learning Rate Optimization of the Backpropagation Algorithm, IEEE Transactions of Neural Networks, Vol. 6, No. 3, pp. 669-677

[13] K. M. Passino, S. Yurkovich, 1998, "Fuzzy Control", Addison Wesley Longman, Inc., ISBN 0-201-18074$\mathrm{X}$

[14] M. Schmidt, T. Stidsen, 1997, "Hybrid Systems: Genetic Algorithms, Neural Networks, and Fuzzy Logic", Diami IR, ISSN 0106-9969

[15] A. F. Naumov, M. Yu. Loktev, I. R. Guralnik and G. Vdovin, 1998, Liquid crystal adaptive lenses with modal control, Optics Letters, Vol. 23, No. 13, pp992994 